Check for updates

Cite this: J. Mater. Chem. C, 2020 8, 15416

Received 2nd July 2020

Accepted 10th September 2020

DOI: $10.1039 / \mathrm{dOtc03139a}$

rsc.li/materials-c

\section{In silico design of 2D polymers containing truxene-based platforms: insights into their structural and electronic properties $\dagger$}

\author{
Sergio Gámez-Valenzuela, ${ }^{a}$ Marcelo Echeverri, (DD ${ }^{\mathrm{b}}$ Berta Gómez-Lor, (D) *b \\ José I. Martínez (D)*b and M. Carmen Ruiz Delgado (D)*a
}

\begin{abstract}
In the present work, we investigate two-dimensional (2D) conjugated polymers based on $\mathrm{C}_{3}$-symmetric truxene-based cores at the density functional theory (DFT) level. In total, 27 different 2D polymers have been exhaustively studied with the aim to explore the impact of the following effects on the electronic and charge-transport properties: (i) the nature of the conjugated platform, going from electron-rich truxene (Tx) and triindole (Tr) units to electron-deficient truxenone (To) cores, (ii) the spacing of the cores with different bridges, i.e., phenylene $(\mathbf{P h})$ or ethynylene (A) units, (iii) the linker position (2,7,12-substitution in the $\mathbf{T}_{\mathbf{2}}$ polymers and 3,8,13-substitution in $\mathbf{T}_{\mathbf{3}}$ polymers), and (iv) the increased number of $\pi$-bridges connecting the cores, from three linkers in $T_{2}$ and $T_{3}$ to six linkers in $T_{2,3}$. To this end, we have carried out a large battery of DFT calculations on fragments extracted from the 2D polymers (dimers and trimers) as well as on the corresponding periodic 2D structures (infinite monolayers and self-assembled monolayers) using periodic boundary conditions. Our results show that simultaneous manipulation of the pore surface size and band-gap engineering together with chargetransport parameters can be achieved in these truxene-based 2D polymers by fine-tuning their structural features. The contributions of this study to the overall understanding of the structure-electronic property relationships of these semiconducting polymers and its correlation with available experimental work are highlighted. Our results provide interesting guidelines to design novel 2D materials with applications ranging from sensing to photocatalysis or electronics.
\end{abstract}

\section{Introduction}

After three decades of enormous research effort, the field of organic semiconductors is currently a mature workbench that has already reached the market. ${ }^{1-3}$ Advances in this area have been closely related to the continuous improvement in the ability of organic semiconductors to transport charge carriers (holes or electrons), which is quantified by their charge carrier mobility $\mu$. While the highest mobilities are usually observed in crystalline or liquid crystalline materials, in the pursuit of an optimal balance between mobility and processability, semiconducting polymers are the preferred candidates. ${ }^{4}$ These

\footnotetext{
${ }^{a}$ Department of Physical Chemistry, University of Malaga, Campus de Teatinos $s / n$, 29071, Malaga, Spain. E-mail: carmenrd@uma.es

${ }^{b}$ Instituto de Ciencia de Materiales de Madrid, CSIC. C/Sor Juana Inés de la Cruz 3, Cantoblanco, Madrid 28049, Spain. E-mail: bgl@icmm.csic.es, joseignacio.martinez@icmm.csic.es

$\dagger$ Electronic supplementary information (ESI) available: Theoretical methods and computational details; chemical structures of the molecular fragments studied theoretically and Study of 2D conjugated polymer networks. See DOI: 10.1039/ dotc03139a
}

materials are usually constituted by conjugated one-dimensional (1D) chains and offer continuous electronic delocalization along the chain axis, and favor the efficient one-dimensional migration of charge carriers. However, hopping of charge carriers between chains is significantly hindered, thus resulting in slow down.

Expanding the $\pi$-conjugation has emerged as an efficient strategy to address the aforementioned drawback; this expansion permits the achievement of equally efficient electron delocalization in two dimension (2D) or three dimension (3D). This possibility has focused increasing attention on $2 \mathrm{D}$ organic polymers as semiconducting materials with enhanced transport dimensionality. ${ }^{5}$

2D covalent organic frameworks (COFs) are porous crystalline solids obtained by the polymerization of organic building blocks functionalized with reactive groups that predetermine their growth along two or more directions through dynamic covalent chemistry. ${ }^{6,7}$ This design strategy gives rise to welldefined, high-surface-area materials whose final properties, topologies and porosities can be conveniently tuned by the choice of monomers and linking groups and by the position of the reactive groups, which determines the growth direction. 
Achieving crystalline order in COFs relies on the reversibility of the chemical reactions used to connect the constituting molecular units via an error-correction mechanism. ${ }^{8}$ Among the small number of chemical reactions that are sufficiently dynamic to enable the synthesis of COFs, Schiff base (imine) formation is the only chemical transformation that gives rise to $\pi$-conjugated structures. ${ }^{9}$ However, imine linkages are far from ideal to induce efficient $\pi$-delocalization between connected units owing to their high polarization. ${ }^{10,11}$ In order to facilitate exciton migration and carrier transportation across these frameworks, efforts are being focused on the search for linkages that promote $\pi$-electron delocalization. Interestingly, the choice of planar $\pi$-conjugated monomers has allowed the synthesis of 2D conjugated microporous polymers (CMPs), even in irreversible coupling conditions, by performing the coupling reactions on the surface ${ }^{12-14}$ or in confined liquid-liquid interfaces. ${ }^{10,15,16}$ This strategy has demonstrated crystalline sheet-like organic frameworks with building units directly linked or connected through bridges and are prone to efficient electronic delocalization (diyne, phenyl, alkyne, etc.). ${ }^{17}$

Our group has long been involved in the development of high-mobility self-assembling molecular materials based on different heptacyclic truxene-related platforms. Truxene can be considered as a 1,3,5-triphenylbenzene planarized through three bridging methylene groups, giving rise to a $\pi$-conjugated heptacyclic system with three additional fused 5-member rings. Varying the nature of the bridging groups (i.e., changing methylene units to amino or ketone units) allows us to obtain molecules that share the truxene-like geometry but show completely different redox and semiconducting behaviors. Thus, while triindole ${ }^{18,19}$ and truxene $e^{20}$ are p-type semiconductors, truxenone ${ }^{21}$ behaves as a high-mobility n-type semiconductor.

Because of their flat trigonal topology, these molecules have recently aroused interest as building units in the construction of functional COFs and CMPs. In fact, recently, microporous polymers based on these semiconducting monomers have been reported, with photocatalytic properties, ${ }^{22-24}$ sensing ability, ${ }^{25,26}$ thermoelectric, ${ }^{27}$ supercapacitor behavior ${ }^{28}$ or field effect behavior. ${ }^{16}$ Optimizing materials for these applications requires the fine-tuning of different parameters, such as energy levels, $\pi$-conjugation or exciton/charge transport. In this sense, theoretical calculations are found to be very useful to predict or rationalize the relationships between chemical structural modifications and the properties of the resulting materials. DFT calculations on the electronic structures of different 2D conjugated polymers have been previously reported, with an emphasis on the role played by the network topology, ${ }^{29-31}$ lattice symmetry ${ }^{32}$ or structural differences between $1 \mathrm{D}$ and $2 \mathrm{D},{ }^{33-35}$ among other structural factors. ${ }^{36-39}$ However, while isolated truxene-based molecules have been amply theoretically explored, a systematic study of their $2 \mathrm{D}$ conjugated polymers has not yet been reported. ${ }^{40-42}$

In this work, we performed an exhaustive investigation of truxene-based 2D conjugated polymers with the aim to explore their chemical structure-electronic property relationships to guide the design of novel optimized 2D materials. To this end, a)

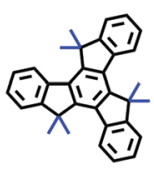

Truxene

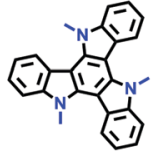

Triindole

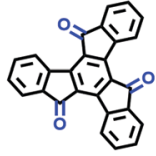

Truxenone

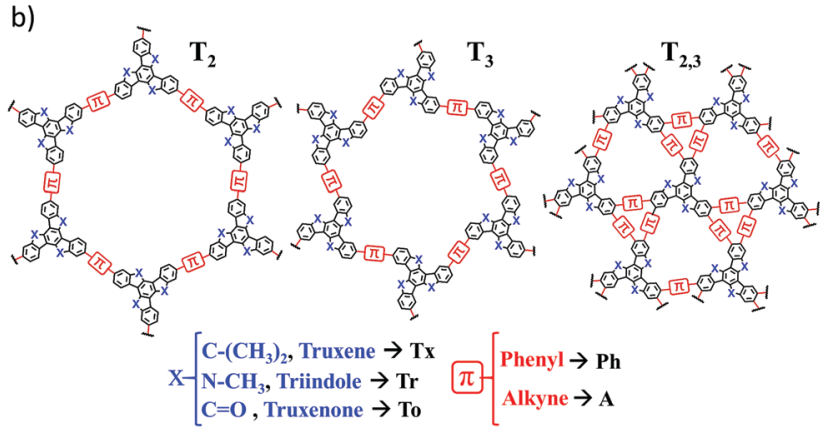

Fig. 1 General chemical structures of the conjugated cores (a) and 2D polymers (b) examined in this study with the notation used throughout the text. Note that $\mathbf{T}_{\mathbf{2}}$ and $\mathbf{T}_{\mathbf{3}}$ polymers refer to tri-substituted materials with different linkage positions $\left(2,7,12\right.$ in $T_{2}$ and $3,8,13$ in $\left.T_{3}\right)$, while $T_{2,3}$ corresponds to hexa-substituted materials in the 2,3,7,8,12 and 13 positions.

we combined an oligomer approach with periodic boundary conditions (PBC) calculations for the 2D lattices. Our selection of truxene-based 2D polymers for this work is shown in Fig. 1. A total of 27 different $2 \mathrm{D}$ polymers were investigated with the ultimate goal of exploring how their electronic and chargetransport properties can be tuned by engineering the organic building blocks (i.e., by changing the nature of the core from $\mathbf{T x}$ to $\mathbf{T r}$ or To or by using different $\pi$-bridges, from phenylene to alkyne) and the connecting mode (i.e., by changing the linkage position from $\mathbf{T}_{2}$ to $\mathbf{T}_{3}$ or by increasing the number of $\pi$-bridges from three units in $\mathbf{T}_{\mathbf{2}}$ and $\mathbf{T}_{\mathbf{3}}$ to six units in $\mathbf{T}_{2,3}$ ). Among the selected 2D polymers, four truxene-based (i.e., $\mathbf{T x}_{\mathbf{2}}, \mathbf{T} \mathbf{T x}_{\mathbf{3}}, \mathbf{P h}-\mathbf{T x}_{\mathbf{2}}$ and $\left.\mathbf{P h}-\mathbf{T x}_{3}\right)^{25}$ and one triindole-based ( $\left.\mathbf{P h}-\mathbf{T r}_{2}\right)$ polymer have been successfully reported ${ }^{16}$ and showed promising applications in sensing and electronics, respectively. We anticipate that this class of truxene-based 2D conjugated networks are potential versatile semiconducting materials for optoelectronic applications.

It is worth noting that most of the first-principles theoretical frameworks adopted here have been very recently used to rationalize similar compounds, such as the first example of a solidstate fluorescent $2 \mathrm{D}-\mathrm{COF}$ bonded via imine linkage ${ }^{37}$ or highlyefficient metal-free COF catalysts towards the oxygen reduction reaction and hydrogen evolution processes. ${ }^{43}$ Taking advantage of this not-yet-fully-harvested theoretical workbench, this exhaustive and systematic study provides a powerful protocol for prefiltering of novel candidate materials towards different potential application fields according to the targeted functionality.

\section{Results and discussion}

The structural and electronic properties of $2 \mathrm{D}$ conjugated polymers containing truxene-based platforms were theoretically investigated 
using a canonical bottom-up approach. First, molecular equivalent fragments extracted from the 2D polymeric structures were investigated in order to evaluate the impact produced by the nature of the core as well as the influence of the nature, number and linkage positions of the bridging connectors on the electronic communication between the conjugated $C_{3}$ symmetric platforms. To this end, we focused on the relationships between the HOMO-LUMO gaps, topologies and structural reorganization energies for hole and electron transport and the chemical structures of these 2D materials. Second, these molecular fragments were then sequentially assembled to form larger subsystems, and truxene-based 2D lattices were generated. The structural features (pore size, geometry of the monolayer, interlayer stacking) and electronic band structures of these 2D COFs were investigated in detail. This study allowed us to explore the pore surface size and band-gap engineering of these promising $2 \mathrm{D}$ materials and their potential as chargetransport materials, with important implications for their application in fields ranging from electronics to photocatalysis or sensing.

\section{Molecular fragments}

We first focused on performing DFT calculations for the molecular fragments of the 2D polymers under study (Fig. S1 and S2, ESI $\dagger$ ). Note that it has been recently demonstrated that calculations of fragments of molecules provide important information about the molecular and electronic structures of 2D conjugated networks. ${ }^{44-50}$

1.1 Structural features. Fig. 2a displays the optimized ground-state structures for the molecular constituents of the $\mathbf{T}_{2}$ polymers. As we can observe, systematic similar torsions $\left(\sim 36^{\circ}\right)$ are found when the cores are linked through a phenylene-bridged unit or when they are directly connected, while coplanar structures are found in alkyne-linked fragments.

Interestingly, the nature of the $C_{3}$-symmetric conjugated platforms plays a key role in their internal disorder: (i) in the truxene (Tx) cores, each methyl group is oriented out of the plane of the conjugated platform and therefore does not sterically hinder the planarity of the overall system, (ii) the trivalent nature of the nitrogen atoms of the triindole ( $\mathbf{T r})$ core results in a moderate distortion of the platform due to the steric hindrance caused by the only existing methyl groups and the outermost benzene rings, and (iii) the truxenone (To) cores are totally coplanar as a consequence of the favorable intramolecular interactions between the $\mathrm{C}=\mathrm{O}$ groups and adjacent hydrogen atoms of the outermost benzene rings (with $\mathrm{C}=\mathrm{O} \cdots \mathrm{H}$ distances of $2.12 \AA)$. The change of the linkage position from para $(2,7,12)$ connections in $\mathbf{T}_{2}$ to meta $(3,8,13)$ connections in $\mathbf{T}_{3}$ yields very similar geometrical distortions (Fig. S4, ESI $\dagger$ ). Importantly, an increase of the number of peripheral linkers from three to six units in $\mathbf{T}_{2,3}$ results in more extended and coplanar skeletons when the cores are directly linked due to the formation of fully fused benzene rings between the platforms or when alkyne groups are connecting the cores (see Fig. 2b). However, in the hexaphenyl-substituted $\mathbf{P h}-\mathbf{T}_{2,3}$ polymers, three-dimensional cyclic cavities are created
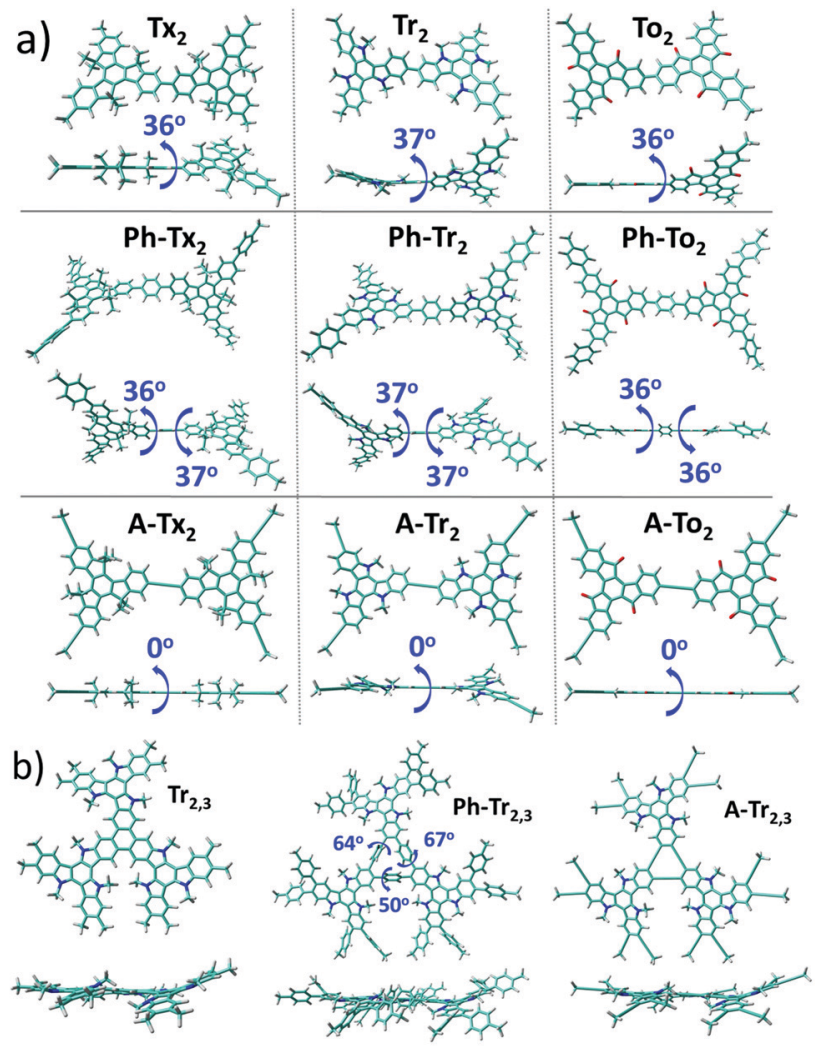

Fig. 2 Top and lateral views of the DFT-optimized structures (PBEO/ $6-31 G^{* *}$ level) for the (a) $\mathbf{T}_{2}$ dimeric models (see Fig. S3a (ESI $\dagger$ ) for the $\mathbf{T}_{3}$ systems) and (b) $\operatorname{Tr}_{2,3}$ trimeric models chosen as representatives for the series of the hexa-substituted $\mathbf{T}_{2,3}$ systems under study (see Fig. S3b (ESI $\dagger$ ) for the rest of the $\mathbf{T}_{2,3}$ systems). The dihedral angles between the conjugated cores and the $\pi$-bridges (in absolute values) are also shown.

between the three external phenylene units connecting the cores that are significantly distorted by $\sim 50-67^{\circ}$ (see $\mathbf{P h}-\mathbf{T r}_{2,3}$ taken as a model in Fig. 2b).

1.2 Electronic features. Fig. 3a shows the frontier molecular orbital energies for the $\mathbf{T}_{2}$ and $\mathbf{T}_{\mathbf{3}}$ fragment models. For the sake of comparison, the influences of the nature of the core, the $\pi$-bridges and the different linkage positions were analyzed separately: (i) both the HOMO and LUMO levels are destabilized (stabilized) on going from the $\mathbf{T x}$ to the $\mathbf{T r}$ or $\mathbf{T o}$ systems; this effect is more pronounced in the case of the HOMO (LUMO), in accordance with the electron-donating and electron-withdrawing character of the $\operatorname{Tr}$ and To units, respectively. This results in a HOMO-LUMO gap decrease in the following order: $\mathbf{T x}>\mathbf{T r}>\mathbf{T o}$, which can be rationalized in terms of the extension of the $\pi$-conjugation (Fig. 3b). As evidenced by the frontier molecular orbitals of the directly connected systems, the HOMOs and LUMOs of the Tx-based systems spread over the central rings along the CC backbone; however, significant contributions from the nitrogen atoms were also found in the HOMOs of the Tr-based systems, while the LUMOs of the To-based systems also spread over more internal ketone groups (see Fig. 3b). Note that similar observations have been previously reported for the isolated trigonal building units. ${ }^{40-42}$ (ii) Considering the effects of the $\pi$-bridge, 

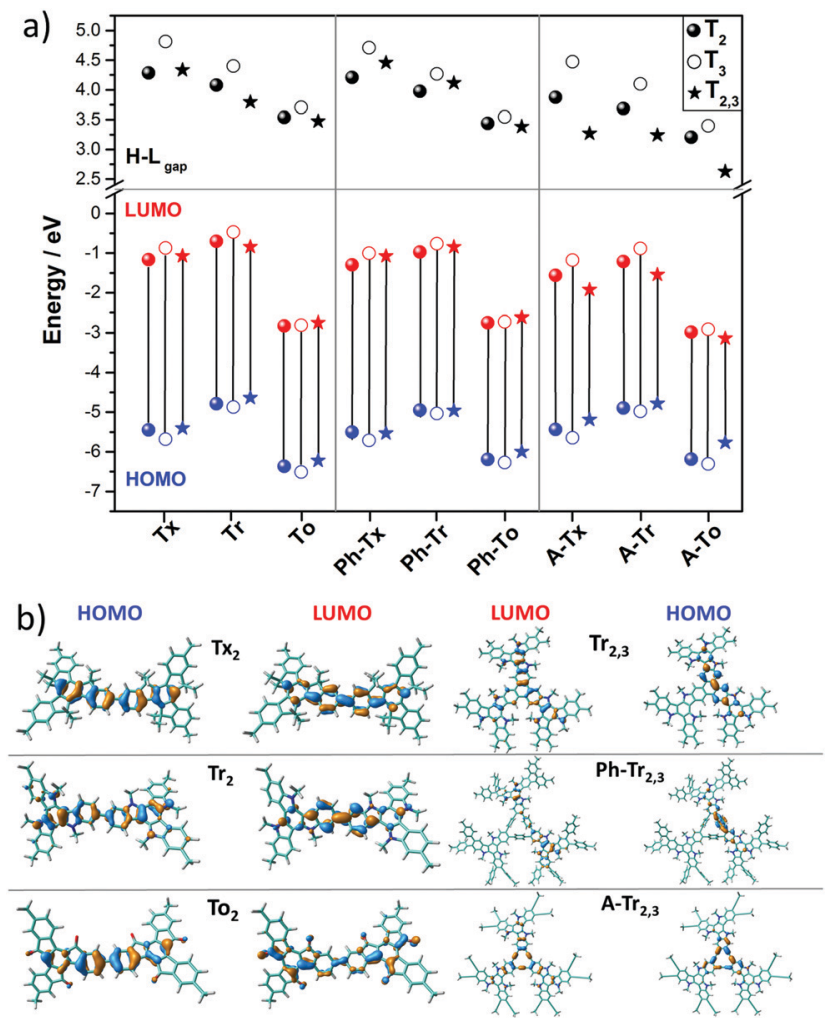

Fig. 3 (a) DFT-calculated (PBEO/6-31G** level) HOMO-LUMO gaps (a) and frontier molecular orbital energies (b) for the $\mathbf{T}_{\mathbf{2}}$ (solid circles), $\mathbf{T}_{\mathbf{3}}$ (open circles) and $\mathbf{T}_{2,3}$ (solid triangle) models. (b) HOMO and LUMO topologies of the directly connected $T_{2}$ dimeric models and $\operatorname{Tr}_{2,3}$ trimeric models chosen as representatives (see Fig. S6 $(\mathrm{ESI} \dagger)$ for the remaining compounds).

the insertion of a phenylene spacer between the cores exerts a slight impact on the HOMO and LUMO energy levels when compared to the directly connected systems, suggesting a minor influence on the $\pi$-electron delocalization between platforms. This is in consonance with the similar distortions found for both the directly connected and phenylene-bridged systems. Conversely, the presence of ethylene linkers produces a significant decrease of the HOMO-LUMO gap, which is associated with moderate stabilization of the LUMO, whereas the HOMO level is slightly affected. Note that the HOMO and LUMO wavefunctions of the alkyne-substituted systems show strong participation of the bridge in the overall $\pi$-conjugation, whereas the contribution of the spacer in their phenylenelinked analogues is less pronounced (see the ESI $\dagger$ ). This indicates more efficient electronic $\pi$-conjugation in the alkyne-substituted systems, in agreement with the electronaccepting nature and the cylindrical symmetry of the alkyne group. (iii) A moderate increase of the HOMO-LUMO gaps was observed when varying the linkage position from $\mathbf{T}_{2}$ to $\mathbf{T}_{3}$ due to the similar LUMO destabilization and HOMO stabilization. This is in line with our previously reported study on Tx-based porous polymers, ${ }^{25}$ where we demonstrated that polymers with truxene cores covalently linked in para positions are better conjugated than their meta-substituted isomers. A comparison of the molecular orbitals for the $\mathbf{T}_{\mathbf{2}}$ and $\mathbf{T}_{\mathbf{3}}$ models (Fig. S6, ESI $\dagger$ ) shows that the connecting conjugated units at the meta positions allow for shorter pathways of alternating single and double bonds, thus providing a less efficient conjugation pathway than the units at the para positions. (iv) Finally, increasing the number of connecting groups has a mixed influence on the energy of the frontier energy levels. Thus, while the HOMO/ LUMO energies are barely affected when comparing the directly connected $\mathbf{T}_{\mathbf{2}}$ and $\mathbf{T}_{\mathbf{2}, 3}$ systems, which show similar HOMO and LUMO wave functions and delocalization over the same number of adjacent rings (for example, compare the HOMO and LUMO orbitals for $\mathbf{T r}_{2}$ and $\mathbf{T r}_{2,3}$ taken as models in Fig. $3 \mathrm{~b}$ ), the presence of six connecting phenylene units results in moderately larger HOMO-LUMO gaps. This can be ascribed to the distorted configuration of the phenyl groups $\left(\sim 60^{\circ}\right.$, as shown in Fig. 2b). In contrast, increasing the number of alkyne bridges from three to six results in lower HOMO-LUMO gaps, indicating better conjugation between the cores, as supported by the more extended electronic delocalization in the HOMO and LUMO orbitals (i.e., see the orbitals for $\mathbf{A}-\mathbf{T r}_{2,3}$ in Fig. $3 \mathrm{~b}$ ).

The nucleus-independent chemical shift (NICS) values for the $\mathbf{T}_{\mathbf{2}}$ and $\mathbf{T}_{\mathbf{3}}$ fragment models were calculated at the geometrical centers of all the phenyl rings (NICS(0)) and at $1 \AA$ above (NICS(1)); see Fig. S8 and S9 (ESI †). The NICS methodology is a widely employed indicator of chemical aromaticity; ${ }^{51,52}$ the more negative the NICS value, the more aromatic the system. On the basis of the calculated data, the aromaticity of these systems is significantly affected by the nature of the core but is barely influenced by the linker units or linkage position. When compared to the reference Tx-based derivatives: (i) the presence of electron-rich Tr platforms results in more negative NICS values (around $2 \mathrm{ppm}$ ), suggesting that the platforms become more aromatic. (ii) The insertion of To cores leads to less negative NICS values, and this effect is more important in the innermost benzene rings (with NICS(0) values of around $-1.2 \mathrm{ppm}$ in the To-based systems compared to values of around $-6.8 \mathrm{ppm}$ for the Tx-based systems); this can be explained in terms of the electron-withdrawing character of the ketone groups, which decreases the aromaticity of the structure in consonance with better electronic delocalization, as previously pointed out by their lowest HOMO-LUMO gap values in the series.

The intramolecular reorganization energy is one of the key molecular parameters governing charge transport, ${ }^{53}$ and it reveals the geometrical adjustments necessary to accommodate a positive or a negative charge. The smaller the value of $\lambda$, the larger the expected charge mobility. With this in mind, we analyzed the effects of the electronic nature of the conjugated platforms on the intramolecular reorganization energies associated with hole $\left(\lambda_{\mathrm{h}}\right)$ and electron $\left(\lambda_{\mathrm{e}}\right)$ transfer. Fig. 4 shows the computed $\lambda$ values for the $\mathbf{T}_{2}$ and $\mathbf{T}_{3}$ dimeric models. The calculated $\lambda_{\mathrm{h}}\left[\lambda_{\mathrm{e}}\right]$ values are in the range of 71-217 [102-369] meV. Note that these values are on the same order of those previously obtained for 2D COF fragments based on pyrene (with $\lambda_{\mathrm{h}}=134 \mathrm{meV}$ and $\lambda_{\mathrm{e}}=192 \mathrm{meV}$ ) or porphyrin (with $\lambda_{\mathrm{h}}=54 \mathrm{meV}$ and $\lambda_{\mathrm{e}}=170 \mathrm{meV}$ ) cores for which high charge-carrier mobility values were predicted. ${ }^{49}$ Among the series, the Tr-based and 


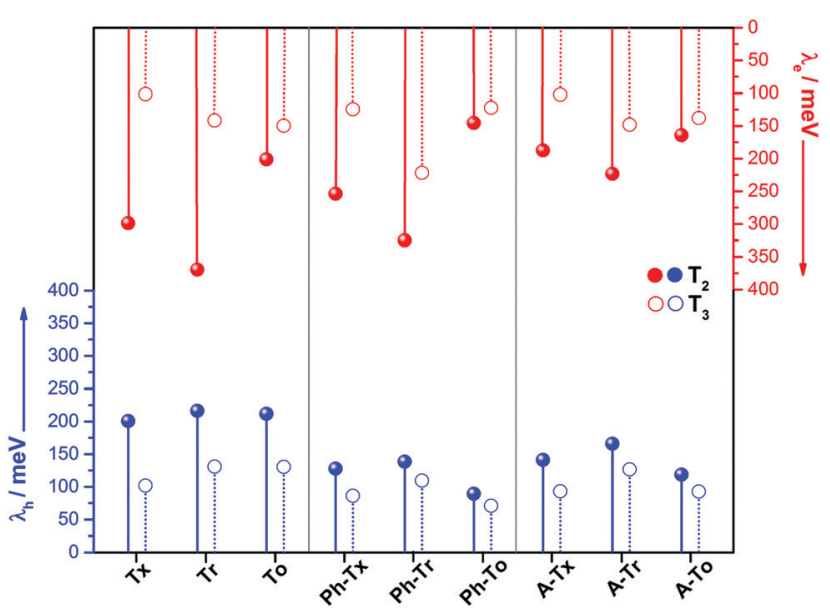

Fig. 4 DFT-calculated (PBE0/6-31G** level) hole (red) and electron (blue) reorganization energies for the $\mathbf{T}_{\mathbf{2}}$ (solid circles) and $\mathbf{T}_{\mathbf{3}}$ (open circles) dimeric models.

To-based systems exhibit the largest and lowest $\lambda$ values, respectively; this can be attributed to the large distortion found in $\mathbf{T r}$ platforms due to the steric hindrance between the $N$-methyl groups and the outermost benzene rings, whereas the To cores exhibit totally coplanar skeletons, allowing for better geometrical relaxation upon charging. The insertion of $\pi$-bridges results in smaller $\lambda$ values, especially for alkyne groups. For instance, linking the cores with phenyl [alkyne] groups results in $\lambda_{\mathrm{h}}$ and $\lambda_{\mathrm{e}}$ decreases of 123 [56] and 93 [37] meV, respectively, for the $\mathbf{T o}_{2}$ derivatives; this behavior can be related to the previously observed enhancement of the $\pi$-electron delocalization between the heptacyclic platforms and to the overall relaxation of the structure upon core elongation. Finally, the structural reorganization needed to accommodate both positive and negative charges decreases by an average of $30 \%$ and $40 \%$, respectively, when the linkage position changes from $\mathbf{T}_{\mathbf{2}}$ to $\mathbf{T}_{3}$; this effect is more pronounced when the cores are directly linked.

\section{2D conjugated polymer networks}

We next investigated the electronic structures of single-layer truxene-based $2 \mathrm{D}$ polymers built by sequentially assembling the previously studied molecular fragments. For all the materials under study, honeycomb-Kagome-like hexagonal lattices were observed, where within the primitive unit cell, the connection between the conjugated platforms exhibits an arrangement of a kagome sublattice while the centers of the cores form a honeycomb sublattice. ${ }^{54}$

2.1 Structural characterization of single-layer 2D COFs. The analysis of the optimized geometries of the $\mathbf{T}_{2}, \mathbf{T}_{3}$ and $\mathbf{T}_{2,3}$ COFs revealed that pore surface engineering of these materials can be achieved by suitable structural design (see Fig. 5 and 6). Whereas the $\mathbf{T}_{2}$ and $\mathbf{T}_{3}$ COFs lead to hexagonal mesopores (accessible pore diameters up to $26 \AA$ ), the $\mathbf{T}_{2,3}$ COFs bear two different kinds of micropores: hexagonal (accessible pore diameter of $\sim 6-11 \AA$ ) and triangular (accessible pore diameter of $\sim 4-6 \AA$ ). Interestingly, the size of the hexagonal mesopores can also be modulated by the insertion of $\pi$-bridges between the cores (i.e., increases of 8 and $4 \AA$, respectively, were found in $\mathbf{T}_{\mathbf{2}}$ and $\mathbf{T}_{\mathbf{3}}$ when comparing the phenylene- or alkyne-based COFs with their directly linked homologues) or by changing the linkage position (i.e., the pore size decreases by $\sim 5 \AA$ when going from para-connected $\mathbf{T}_{2}$ to meta-connected $\mathbf{T}_{3}$ ). The wide range of pore sizes and pore geometries should bring novel functionalities to these materials, such as multiselectivity, that can be exploited in versatile applications such as adsorption and separation science. It is interesting to remark that surface pore engineering is currently attracting paramount interest. $^{55-57}$

In accordance with the optimized molecular fragments, the optimized periodic single layers predict that the Tx- and To-based COF structures are planar, while the Tr-homologues have a moderately distorted configuration as a consequence of the steric repulsions between the $N$-methyl groups and the hydrogen atoms of the adjacent phenyl groups. This structural feature plays a role in the electronic total charge distribution of these 2D materials. Fig. 5 and 6 depict colour total electronic density maps (increasing charge density from the light green to the red/purple regions) associated with the cutting-planes of the 3D total charge isodensity, which becomes slightly inhomogeneous in specific regions of some of the compounds due to out-of-plane distortions of the structures. The distorted configuration of the Tr-based COF monolayer will have a strong implication for the preferential interlayered bulk stacking, as discussed below.

2.2 Electronic structures of the single-layer 2D COFs. We next examined the electronic band structures of the $2 \mathrm{D}$ polymers under study. One interesting finding that can be extracted from the band structures is that these systems can be classified as semiconducting 2D materials with band-gaps in the range of 1.17-3.13 eV (Fig. 7); therefore, they are very interesting for photovoltaic applications according to the Shockley-Queisser limit. ${ }^{58}$ In good analogy with the HOMO-LUMO gap trends calculated for the fragment models, the following observations can be pointed out: (i) a decrease of the band-gap occurs when going from the $\mathbf{T x}$ and $\mathbf{T r}$ cores to the To core (i.e., from 2.37, 2.22 and $1.77 \mathrm{eV}$ going from $\mathbf{T x}_{2}$ and $\mathbf{T r}_{2}$ to $\mathbf{T o}_{2}$ ). (ii) A moderate bandgap increase was found when changing the linkage

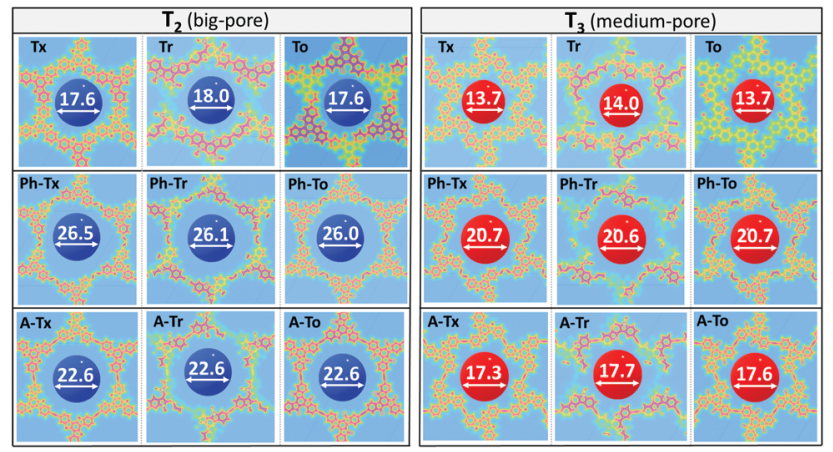

Fig. 5 DFT-PBE-calculated surface charge density colour maps in the $x y$ plane (at the $z$-position) for all the $T_{2}$ and $T_{3} 2 D$ COFs under study. Accessible pore diameter $(\AA)$ values are also shown. 


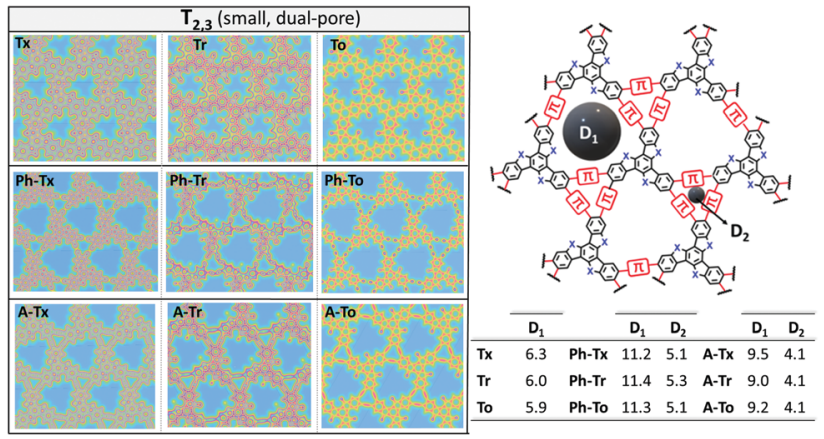

Fig. 6 DFT-PBE-calculated surface charge density colour maps in the $x y$ plane (at the $z$-position) for the $T_{2,3} 2 \mathrm{D}$ COFs under study. Accessible pore diameter (Å) values for the hexagonal (D1) and trigonal (D2) pores are also shown.
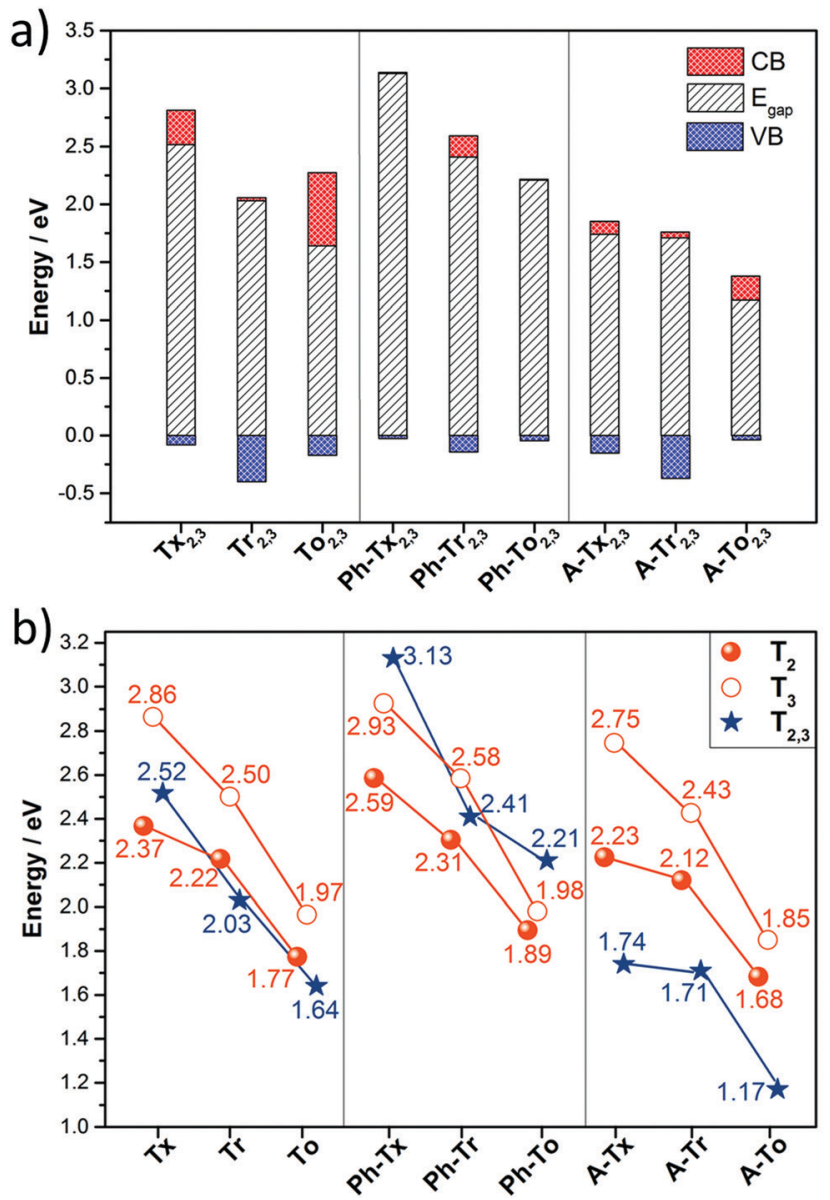

Fig. 7 (a) Calculated band-gaps and bandwidths for the conduction bands (CBs) and valence bands (VBs) of all the hexa-substituted truxenebased 2D COFs. (b) Comparison of the calculated band-gaps for all the 2D polymers under study. The zero energy is taken to correspond to the valence band maximum.

position from the para to the meta connection, suggesting more effective conjugation pathways in the $\mathbf{T}_{\mathbf{2}}$ COFs compared to the $\mathbf{T}_{3}$ COFs. (iii) The insertion of phenylene spacers slightly alter the bandgaps in $\mathbf{T}_{\mathbf{2}}$ and $\mathbf{T}_{\mathbf{3}}$ polymers but results in larger band-gaps in the $\mathbf{T}_{2,3}$ polymers (i.e., from 2.50 [2.03] to 2.58 [2.41] eV on going from $\mathbf{T r}_{2}\left[\mathbf{T r}_{2,3}\right]$ to $\mathbf{P h}-\mathbf{T r}_{2}\left[\mathbf{P h}-\mathbf{T r}_{2,3}\right]$, respectively), which can be ascribed to the more twisted structures of the latter, thus decreasing the electronic conjugation between the core. (iv) A significant decrease of the bandgap was found in the alkyne-connected 2D polymers, with the lowest value found for $\mathbf{A}-\mathbf{T o}_{2,3}(1.17 \mathrm{eV})$; that is, the flat truxenone platforms hexasubstituted with alkyne spacers facilitate the extension of the electronic conjugation and enable better electronic communication between the cores.

The DFT-PBE0 band structures of the $\mathbf{T}_{\mathbf{2}}$ and $\mathbf{T}_{2,3}$ COFs are displayed in Fig. 8. The three-armed platforms in the $\mathbf{T}_{2} \mathbf{C O F s}$ lead to the formation of highly symmetric kagome-like lattices,$^{32}$ which results in a totally flat top of the valence band (VB) and bottom of the conduction band (CB); this affords fully localized charge carriers and infinitive carrier effective masses, which translates into vanishing charge carrier mobilities (Fig. 8a). Only a very slight gain of the valence band dispersion is observed when the cores are directly connected through meta connections in $\mathbf{T}_{\mathbf{3}}$ (Fig. S10, ESI $\dagger$ ). The insertion of phenylene or alkyne $\pi$-bridges between the cores retained the totally flat character of the bands around the Fermi level in both the $\mathbf{T}_{\mathbf{2}}$ and $\mathbf{T}_{3}$ COFs (Fig. S10 and S11, ESI $\dagger$ ). These predicted flat bands contrast with the high mobility values experimentally obtained for $\mathbf{P h}-\mathbf{T r}_{2}$ COFs, one of the benchmark hole transport 2D conjugated polymers reported to date. ${ }^{16}$ In this case, the high mobility values probably reflect the favorable electronic coupling between the cores of the adjacent layers, which may increase the bandwidths along the vertical direction. To reinforce this hypothesis, we analyzed the preferential interlayer stacking of different selected 2D COFs (see the Discussion in Section 2.3).

On the other hand, the electronic structures of COFs based on six-armed cores result in more dispersive bands (Fig. 7a and $8 \mathrm{~b}$ ). In this context, it is worth noting that the more dispersive the valence band [conduction band] at the VB maximum [BC minimum], the larger the expected hole [electron] charge carrier mobility. Interestingly, a large variation of the bandwidths was found in the $\mathbf{T}_{2,3}$ COFs (i.e., in the range of $26-400 \mathrm{meV}$ and $6-633 \mathrm{meV}$ for the $\mathrm{CB}$ and $\mathrm{VB}$, respectively). Particularly large $\mathrm{CB}[\mathrm{VB}]$ bandwidths and low effective masses for holes [electrons] were found for the $\mathbf{T r}_{2,3}$ $\left[\mathbf{A}-\mathbf{T o}_{2,3}\right]$ polymers; therefore, these systems are promising hole and electron charge-transport 2D materials, respectively. On the other hand, large bandwidths for both $\mathrm{CB}$ and VB (and low hole and electron effective masses) were obtained for $\mathbf{T o}_{2,3}$, $\mathbf{A}-\mathbf{T x}_{\mathbf{2}, 3}$ and $\mathbf{A}-\mathbf{T r}_{2,3}$; therefore, they are attractive ambipolar charge carrier materials (see Fig. 8b for the directly connected $\mathbf{T}_{2,3}$ COFs and Fig. S12 (ESI $\dagger$ ) for the alkyne-substituted $\mathbf{A}-\mathbf{T}_{2,3} \mathrm{COFs}$ ).

At this point, it is interesting to remark that all the compounds analyzed in this study mainly exhibit $\pi$-type semiconducting character, with the Fermi energy almost pinning the valence band in some cases. The 3D isosurfaces of the orbital electronic densities corresponding to the valence and conduction bands of the different extended 2D COF systems studied here provide 


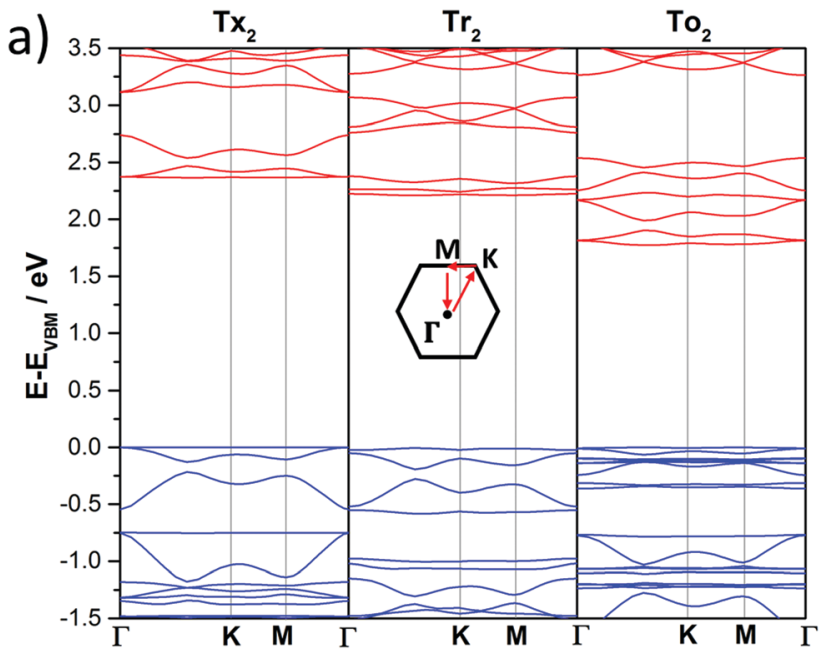

b)

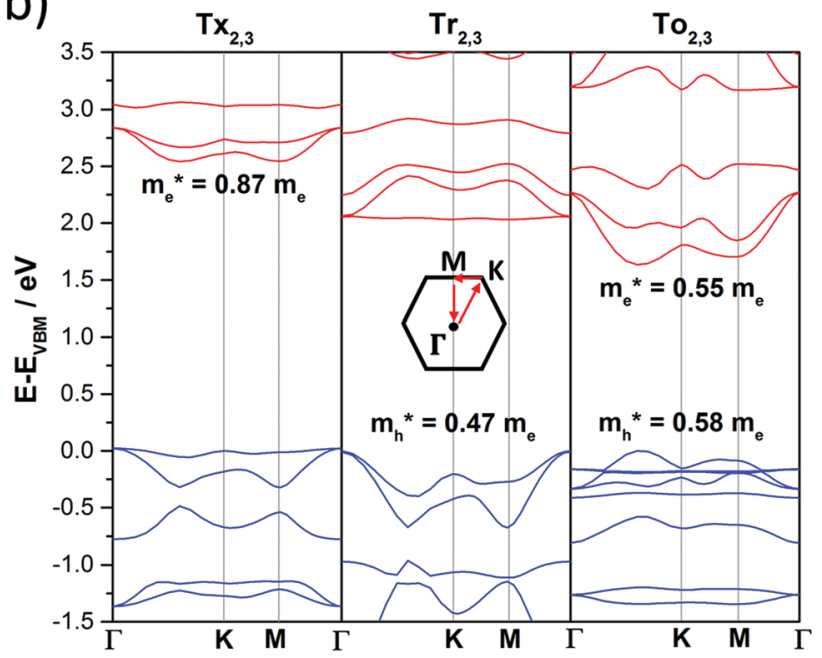

Fig. 8 DFT-PBE-calculated electronic band structures of directly coreconnected 2D polymers: $\mathbf{T x}_{2}, \mathrm{Tr}_{\mathbf{2}}$ and $\mathrm{To}_{\mathbf{2}}$ (a) and $\mathrm{Tx}_{2,3}, \mathrm{Tr}_{2,3}$ and $\mathrm{To}_{2,3}$ (b). The valence and conduction bands are marked in blue and red, respectively. The zero energy is taken to correspond to the valence band maximum, $E_{\mathrm{VBM}}$, while the $x$-axis labels denote a path through the 3D space of the $k$-vectors. Points of high symmetry in the Brillouin zone are labeled as $\Gamma(0,0,0), M(0,1 / 2,0)$ and $K(1 / 3,2 / 3,0)$, all in reciprocal space coordinates. For the electronic band structures of the remaining $2 \mathrm{D}$ polymers under study, see Fig. S10-S13 (ESI $\dagger$ ).

valuable information about the spatial localization and delocalization degrees of these states, the homogeneity in the spatial distribution of the states, and the preferential carrier transport paths across the compounds (Fig. S13-S16, ESI $\dagger$ ). For instance, the engineered $\mathbf{A}-\mathbf{T r}_{2,3}$ system, which is predicted to have narrower band-gaps and pronounced electronic $k$-dispersions, exhibits similar morphologies for the valence and conduction bands, with a more delocalized spatial distribution of the valence band (Fig. S16, ESI $\dagger$ ).

The time-dependent DFT-based UV-Vis absorption spectra of the $\mathbf{T}_{2}$ and $\mathbf{T}_{3} 2$ D polymers were also calculated (Fig. S17, ESI $\dagger$ ). A good correlation can be found with the experimental results previously reported for truxene-based $\left(\mathbf{T x}_{\mathbf{2}}, \mathbf{T} \mathbf{T x}_{\mathbf{3}}, \mathbf{P h}-\mathbf{T x}_{\mathbf{2}} \text { and } \mathbf{P h}-\mathbf{T x}_{\mathbf{3}}\right)^{25}$ polymers. Please note that UV-Vis absorption (photoexcitation) spectra are of fundamental importance for applications ranging from sensing to photocatalysis and photovoltaics. We have found that in these systems, it is possible to modulate the absorption spectral response from the UV region to the near infrared region through suitable chemical modification, such as from $325 \mathrm{~nm}$ in $\mathbf{T x}_{\mathbf{3}}$ to $726 \mathrm{~nm}$ in $\mathbf{A}-\mathbf{T} \mathbf{T}_{2}$ (a more detailed explanation can be found in the ESI $\dagger$ ). These differences are also of fundamental interest for their sensing behavior, as we recently demonstrated for some of these truxene-relatedmaterials. $^{25}$ The character of the symmetry of these valence and conduction orbital states (see Fig. S13-S16, ESI $\dagger$ ) will be crucial to yield the permitted optical transitions captured by the computed photoabsorption spectra (see Fig. S17, ESI $\dagger$ ) because the main contribution to the primary electronic excitation in these compounds comes from excitation of an electron from the valence band towards the conduction band (see the ESI $\dagger$ ).

2.3 Preferential interlayer stacking of 2D COFs. In order to check the preferential interlayered bulk-stacking fashion adopted by the different $2 \mathrm{D}$ layers, we computed the infinite crystal-bulk configurations for the alkyne-based $\mathbf{T}_{2}$ and $\mathbf{T}_{3}$ COFs (which showed more pronounced band dispersion in the $2 \mathrm{D}$ layers) in both $\mathrm{AA}$ and $\mathrm{AB}$ stacking modes. The results of these calculations are reflected in Fig. 9. Other intermediate configurations between $\mathrm{AA}$ and $\mathrm{AB}$ stacking were analyzed, yielding unfavourable results in all cases compared with the canonical $\mathrm{AA}$ and $\mathrm{AB}$ stacking modes. It is interesting to note that on average, and as expected, the cohesive energy $\left(E_{\mathrm{c}}\right)$ increases with decreasing inter-layer distance (with $L$ values ranging between 2.21 and $4.38 \AA$ ). We obtained a wide range of cohesive energies for the different systems, going from systems for which subtle emerging inter-layer chemistry turns into significant via potential $\pi-\pi$ stacking or some other type of interlayer
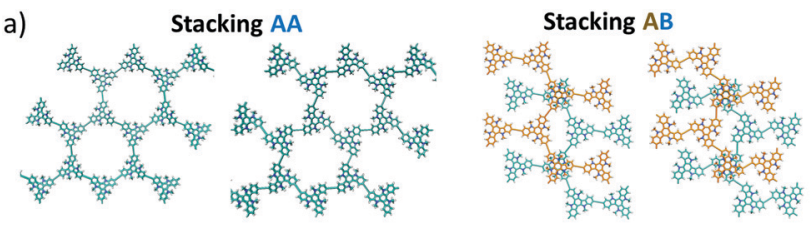

\begin{tabular}{|c|c|c|c|c|}
\hline 2D polymer & Pore & Stacking & $L(\AA ̊)$ & $E_{\mathrm{c}}(\mathrm{kcal} / \mathrm{mol})$ \\
\hline \multirow{2}{*}{ A-Tx ${ }_{2}$} & \multirow{2}{*}{ big } & $A A$ & 3.86 & +87.75 \\
\hline & & $A B$ & 3.22 & -34.20 \\
\hline \multirow{2}{*}{ A- $\mathrm{Tr}_{2}$} & \multirow{2}{*}{ big } & $A A$ & 3.79 & -35.67 \\
\hline & & $A B$ & 2.21 & -58.80 \\
\hline \multirow{2}{*}{$\mathrm{A}-\mathrm{To}_{2}$} & \multirow{2}{*}{ big } & $A A$ & 4.12 & -46.42 \\
\hline & & $A B$ & 4.25 & -24.12 \\
\hline \multirow{2}{*}{ A-Tx $x_{3}$} & \multirow{2}{*}{ medium } & AA & 3.94 & +341.08 \\
\hline & & $A B$ & 3.23 & -36.83 \\
\hline \multirow{2}{*}{ A- $\operatorname{Tr}_{3}$} & \multirow{2}{*}{ medium } & AA & 3.79 & -36.00 \\
\hline & & $A B$ & 2.49 & -67.01 \\
\hline \multirow{2}{*}{$\mathrm{A}-\mathrm{To}_{3}$} & \multirow{2}{*}{ medium } & AA & 4.20 & -33.24 \\
\hline & & $A B$ & 4.38 & -28.07 \\
\hline
\end{tabular}

Fig. 9 (a) Pictorial top views of the two different stacking $A A$ and $A B$ configurations. (b) Computed data of the fully optimized inter-layer stacking distance (in $\AA$ ) and inter-layer cohesive energy (in kcal mol ${ }^{-1}$ ), defined as the difference between the total energy crystal-bulk (with one layer per unit cell) and the isolated single-layer, for the $\mathbf{A}-\mathbf{T}_{\mathbf{2}}$ and $\mathbf{A}-\mathbf{T}_{\mathbf{3}}$ compounds. 
electronic hybridization, such as the case of the A-Tr compounds (cohesive energies in the range of $-65 \mathrm{kcal} \mathrm{mol}^{-1}$ ), and other systems with only electrostatic and vdW interlayer cohesion, such as the A-To compounds (around $-25 \mathrm{kcal} \mathrm{mol}^{-1}$ ). On the other hand, AA stacking of the A-Tx systems leads to unstable configurations due to physical steric impediment towards stacking, which is reflected in their high positive cohesive energies due to the numerical instabilities when the lattice-parameter relaxation algorithm is applied. However, it is worth noting that $\mathrm{AB}$ stacking is the preferential stacking configuration for the A-Tr and A-Tx systems, while the A-To compounds prefer to stack in AA fashion. Interestingly, these results are in good accordance with the available X-ray data reported for triindole ${ }^{18,59,60}$ (truxenone) ${ }^{21}$ cores in selfassembling systems, where a preferential staggered (eclipsed) stacking conformation of the cores is adopted for which large electronic couplings are obtained. This favourable electronic coupling between the cores of the adjacent layers may increase the bandwidth along the vertical direction and probably explains the high carrier mobility of the experimentally obtained $\mathbf{P h}-\mathbf{T r}_{\mathbf{2}}{ }^{16}$ despite the flat bands predicted for the $\mathbf{T}_{\mathbf{2}}$ and $\mathbf{T}_{\mathbf{3}}$ single-layer COFs.

Interestingly, this variety of possible scenarios predicted by our theoretical protocol leads to high versatility in selecting candidates for different applications: going from easy-todelaminate systems, allowing the transfer of a 2D single layer to more interesting inert technological substrates, up to highly robust stacked crystals with potential use in optoelectronic applications or for interpore gas-sensing purposes.

\section{Conclusions}

In summary, we have performed a comprehensive and exhaustive investigation at the DFT level of the structural and electronic features of 27 different 2D conjugated polymer networks based on $C_{3}$-symmetric platforms (i.e., truxene, triindole and truxenone). The conjugated cores are connected directly or through alkyne or phenylene spacers, whose number and linkage position (from para to meta) have also been explored. Semiconductors band-gap values in the range between 1.17 and $3.13 \mathrm{eV}$ are predicted.

Our results show that wide tuning of the structural properties (i.e., geometry or pore size) and electronic properties (i.e. HOMO-LUMO levels, band-gap or band dispersion) can be achieved in these truxene-based $2 \mathrm{D}$ polymers by suitable selection of the trigonal building blocks and the nature and position of the connecting moieties. In the same line, a strong modulation of the absorption spectral response from the UV region to the near infrared region is found through suitable chemical modification. Please note that the above parameters will determine properties such as the selectivity/sensitivity of sensing polymers towards analytes of interest and will dictate their photocatalytic activity.

On the other hand, large bandwidths and low effective masses (and thus, high carrier mobilities) are predicted for $2 \mathrm{D}$ polymers with six-armed triindole and truxenone cores, connected directly $\left(\mathbf{T r}_{2,3}\right.$ and $\left.\mathbf{T o}_{2,3}\right)$ or through alkyne bridges $\left(\mathbf{A}-\mathbf{T r}_{2,3}\right.$ and $\mathbf{A}-\mathbf{T o}_{2,3}$ ). Preferential interlayered stacking is also expected to result in favorable electronic couplings between the cores and may increase the bandwidths along the vertical direction, thus positively affecting the charge-carrier mobilities. This probably explains the high mobility experimentally determined for $\mathbf{P h}-\mathbf{T r}_{2}{ }^{16}$ in spite of the fact that flat bands are predicted for polymers with three-armed cores $\left(\mathbf{T}_{\mathbf{2}}\right.$ and $\left.\mathbf{T}_{\mathbf{3}}\right)$. In fact, the calculations suggest preferred $\mathrm{AB}$ stacking between layers for the related triindole-based polymers, which would lead to a staggered arrangement of neighbouring cores. This organization has been previously demonstrated to result in pronounced electronic coupling and high hole mobilities in triindole self-assembling systems. ${ }^{18,59,60}$ The synthesis of selected candidates (particularly those with monomers connected via six ethynyl linkers) is now actively ongoing in our laboratories in an attempt to obtain high mobility semiconductors and validate our theoretical results.

Overall, our findings shed light on the structure-property relationships of $2 \mathrm{D}$ truxene-based conjugated polymers and provide interesting guidelines to design novel 2D materials with applications ranging from sensing to photocatalysis or electronics. Additionally, this computational first-principlesbased strategy emerges as a powerful tool to achieve in silico pre-filtering of future similar 2D materials, which, by adequate and careful selection of building blocks, will permit the prediction, within the limits of the level of theory employed, of emerging optoelectronic features as a lighthouse to experimentalists according to the desired targeted functionality.

\section{Conflicts of interest}

There are no conflicts to declare.

\section{Acknowledgements}

This work was financially supported by the MINECO (CTQ201678557-R) and MICINN (PID2019-104125RB-I00) of Spain. JIM acknowledges financial support from Spanish MINECO (MAT2017-85089-C2-1-R, RYC-2015-17730), Comunidad de Madrid via Programa de Investigación Tecnologías 2018 (FOTOART-CM S2018/NMT-4367), and the innovation program under grant agreements 785219 and 881603 (GrapheneCore2 and GrapheneCore3-Graphene-based disruptive technologies, respectively). The work at the University of Málaga was funded by the MICINN (PID2019-110305GB-I00) and by the Junta de Andalucia (P09-FQM-4708, UMA18-FEDERJA-080). S. G.-V. thanks the MINECO for a FPU predoctoral fellowship (FPU17/04908). Computer resources, technical expertise and assistance provided by the SCBI (Supercomputing and Bioinformatics) centre of the University of Málaga are gratefully acknowledged.

\section{Notes and references}

1 P. Samorí and V. Palermo, Flexible Carbon-based Electronics, Wiley-VCH, Weinheim, 2018. 
2 H. Klauk, Organic electronics: materials, manufacturing, and applications, Wiley-VCH, Weinheim, 2006.

3 H. Klauk, Organic electronics II: more materials and applications, Wiley-VCH, Weinheim, 2012.

4 S. Fratini, M. Nikolka, A. Salleo, G. Schweicher and H. Sirringhaus, Nat. Mater., 2020, 19, 491-502.

5 D. F. Perepichka and F. Rosei, Science, 2009, 323, 216-217.

6 M. S. Lohse and T. Bein, Adv. Funct. Mater., 2018, 28, 1705553.

7 H. V. Babu, M. G. M. Bai and M. Rajeswara Rao, ACS Appl. Mater. Interfaces, 2019, 11, 11029-11060.

8 Y. Li, W. Chen, G. Xing, D. Jiang and L. Chen, Chem. Soc. Rev., 2020, 49, 2852-2868.

9 J. L. Segura, M. J. Mancheño and F. Zamora, Chem. Soc. Rev., 2016, 45, 5635-5671.

10 D. Zhou, X. Tan, H. Wu, L. Tian and M. Li, Angew. Chem., Int. Ed., 2019, 58, 1376-1381.

11 M. R. Rao, Y. Fang, S. De Feyter and D. F. Perepichka, J. Am. Chem. Soc., 2017, 139, 2421-2427.

12 W. Liu, X. Luo, Y. Bao, Y. P. Liu, G. H. Ning, I. Abdelwahab, L. Li, C. T. Nai, Z. G. Hu, D. Zhao, B. Liu, S. Y. Quek and K. P. Loh, Nat. Chem., 2017, 9, 563-570.

13 L. Cardenas, R. Gutzler, J. Lipton-Duffin, C. Fu, J. L. Brusso, L. E. Dinca, M. Vondráček, Y. Fagot-Revurat, D. Malterre, F. Rosei and D. F. Perepichka, Chem. Sci., 2013, 4, 3263-3268.

14 D. Cui, D. F. Perepichka, J. M. MacLeod and F. Rosei, Chem. Soc. Rev., 2020, 49, 2020-2038.

15 S. Wu, M. Li, H. Phan, D. Wang, T. S. Herng, J. Ding, Z. Lu and J. Wu, Angew. Chem., Int. Ed., 2018, 57, 8007-8011.

16 C. Li, Y. Wang, Y. Zou, X. Zhang, H. Dong and W. Hu, Angew. Chem., Int. Ed., 2020, 59, 9403-9407.

17 C. Ruiz, J. T. López Navarrete, M. C. Ruiz Delgado and B. Gómez-Lor, Org. Lett., 2015, 17, 2258-2261.

18 C. Ruiz, U. K. Pandey, R. Termine, E. M. García-Frutos, G. López-Espejo, R. P. Ortiz, W. Huang, T. J. Marks, A. Facchetti, M. C. Ruiz Delgado, A. Golemme and B. Gómez-Lor, ACS Appl. Mater. Interfaces, 2016, 8, 26964-26971.

19 A. Benito-Hernández, U. K. Pandey, E. Cavero, R. Termine, E. M. García-Frutos, J. L. Serrano, A. Golemme and B. Gómez-Lor, Chem. Mater., 2013, 25, 117-121.

20 K. Q. Zhao, C. Chen, H. Monobe, P. Hu, B. Q. Wang and Y. Shimizu, Chem. Commun., 2011, 47, 6290-6292.

21 S. Gómez-Esteban, A. Benito-Hernandez, R. Termine, G. Hennrich, J. T. L. Navarrete, M. C. Ruiz Delgado, A. Golemme and B. Gómez-Lor, Chem. - Eur. J., 2018, 24, 3576-3583.

22 J. Guadalupe, A. M. Ray, E. M. Maya, B. Gómez-Lor and M. Iglesias, Polym. Chem., 2018, 9, 4585-4595.

23 V. R. Battula, H. Singh, S. Kumar, I. Bala, S. K. Pal and K. Kailasam, ACS Catal., 2018, 8, 6751-6759.

24 A. Valverde-González, C. G. López Calixto, M. Barawi, M. Gomez-Mendoza, V. A. De La Peña O’Shea, M. Liras, B. Gómez-Lor and M. Iglesias, ACS Appl. Energy Mater., 2020, 3, 4411-4420.

25 M. Echeverri, S. Gámez-Valenzuela, R. C. González-Cano, J. Guadalupe, S. Cortijo-Campos, J. T. López Navarrete,
M. Iglesias, M. C. Ruiz Delgado and B. Gómez-Lor, Chem. Mater., 2019, 31, 6971-6978.

26 X. Liu, Y. Xu and D. Jiang, J. Am. Chem. Soc., 2012, 134, 8738-8741.

27 A. E. Sadak, E. Karakuş, Y. M. Chumakov, N. A. Dogan and C. T. Yavuz, ACS Appl. Energy Mater., 2020, 3, 4983-4994.

28 X. C. Li, Y. Zhang, C. Y. Wang, Y. Wan, W. Y. Lai, H. Pang and W. Huang, Chem. Sci., 2017, 8, 2959-2965.

29 A. V. Kuklin, G. V. Baryshnikov, B. F. Minaev, N. Ignatova and H. Ågren, J. Phys. Chem. C, 2018, 122, 22216-22222.

30 Y. Jing and T. Heine, J. Am. Chem. Soc., 2019, 141, 743-747.

31 M. A. Springer, T. J. Liu, A. Kuc and T. Heine, Chem. Soc. Rev., 2020, 49, 2007-2019.

32 S. Thomas, H. Li, C. Zhong, M. Matsumoto, W. R. Dichtel and J. L. Bredas, Chem. Mater., 2019, 31, 3051-3065.

33 R. Gutzler and D. F. Perepichka, J. Am. Chem. Soc., 2013, 135, 16585-16594.

34 J. Wen, D. Luo, L. Cheng, K. Zhao and H. Ma, Macromolecules, 2016, 49, 1305-1312.

35 R. Gutzler and Phys Chem, Chem. Phys., 2016, 18, 29029-29100. 36 D. J. Rizzo, Q. Dai, C. Bronner, G. Veber, B. J. Smith, M. Matsumoto, S. Thomas, G. D. Nguyen, P. R. Forrester, W. Zhao, J. H. Jørgensen, W. R. Dichtel, F. R. Fischer, H. Li, J. L. Bredas and M. F. Crommie, Nano Lett., 2020, 20, 963-970.

37 P. Albacete, J. I. Martínez, X. Li, A. López-Moreno, S. MenaHernando, A. E. Platero-Prats, C. Montoro, K. P. Loh, E. M. Pérez and F. Zamora, J. Am. Chem. Soc., 2018, 140, 12922-12929.

38 P. Zhu and V. Meunier, J. Chem. Phys., 2012, 137, 244703.

39 S. Thomas, H. Li, R. R. Dasari, A. M. Evans, I. Castano, T. G. Allen, O. G. Reid, G. Rumbles, W. R. Dichtel, N. C. Gianneschi, S. R. Marder, V. Coropceanu and J. L. Brédas, Mater. Horiz., 2019, 6, 1868-1876.

40 M. Reig, G. Bagdziunas, A. Ramanavicius, J. Puigdollers and D. Velasco, Phys. Chem. Chem. Phys., 2018, 20, 17889-17898.

41 A. Tripathi and C. Prabhakar, J. Phys. Org. Chem., 2019, 32, e3944.

42 A. Benito-Hernández, M. T. El-Sayed, J. T. López Navarrete, M. C. Ruiz Delgado and B. Gómez-Lor, Org. Chem. Front., 2018, 5, 1748-1755.

43 S. Royuela, E. Martínez-Periñán, M. P. Arrieta, J. I. Martínez, M. M. Ramos, F. Zamora, E. Lorenzo and J. L. Segura, Chem. Commun., 2020, 56, 1267-1270.

44 L. Li, Z. Cai, Q. Wu, W. Y. Lo, N. Zhang, L. X. Chen and L. Yu, J. Am. Chem. Soc., 2016, 138, 7681-7686.

45 G. V. Baryshnikov, B. F. Minaev, N. N. Karaush and V. A. Minaeva, RSC Adv., 2014, 4, 25843-25851.

46 L. Li, W. Y. Lo, Z. Cai, N. Zhang and L. Yu, Macromolecules, 2016, 49, 6903-6909.

47 J. Wen, D. Luo, L. Cheng, K. Zhao and H. Ma, Macromolecules, 2016, 49, 1305-1312.

48 R. Gutzler and D. F. Perepichka, J. Am. Chem. Soc., 2013, 135, 16585-16594.

49 S. Thomas, H. Li, R. R. Dasari, A. M. Evans, I. Castano, T. G. Allen, O. G. Reid, G. Rumbles, W. R. Dichtel, N. C. Gianneschi, 
S. R. Marder, V. Coropceanu and J. L. Brédas, Mater. Horiz., 2019, 6, 1868-1876.

50 S. Jhulki, A. M. Evans, X. L. Hao, M. W. Cooper, C. H. Feriante, J. Leisen, H. Li, D. Lam, M. C. Hersam, S. Barlow, J. L. Brédas, W. R. Dichtel and S. R. Marder, J. Am. Chem. Soc., 2020, 142, 783-791.

51 P. V. R. Schleyer, C. Maerker, A. Dransfeld, H. Jiao and N. J. R. Van Eikema Hommes, J. Am. Chem. Soc., 1996, 118, 6317-6318. 52 T. K. Zywietz, H. Jiao, P. V. R. Schleyer and A. De Meijere, J. Org. Chem., 1998, 63, 3417-3422.

53 V. Coropceanu, J. Cornil, D. A. da Silva Filho, Y. Olivier, R. Silbey and J. L. Brédas, Chem. Rev., 2007, 107, 926-952.

54 C. Barreteau, F. Ducastelle and T. Mallah, J. Phys.: Condens. Matter, 2017, 29, 465302.
55 R. R. Liang, S. Q. Xu, L. Zhang, A. Ru-Han, P. Chen, F. Z. Cui, Q. Y. Qi, J. Sun and X. Zhao, Nat. Commun., 2019, 10, 4609.

56 T. Y. Zhou, S. Q. Xu, Q. Wen, Z. F. Pang and X. Zhao, J. Am. Chem. Soc., 2014, 136, 15885-15888.

57 A. Nagai, Z. Guo, X. Feng, S. Jin, X. Chen, X. Ding and D. Jiang, Nat. Commun., 2011, 2, 536.

58 W. Shockley and H. J. Queisser, J. Appl. Phys., 1961, 32, 510-519.

59 E. M. García-Frutos, E. Gutierrez-Puebla, M. A. Monge, R. Ramírez, P. de Andrés, A. de Andrés, R. Ramírez and B. Gómez-Lor, Org. Electron., 2009, 10, 643-652.

60 E. M. García-Frutos, G. Hennrich, E. Gutierrez, A. Monge and B. Gómez-Lor, J. Org. Chem., 2010, 75, 1070-1076. 